\title{
Taser-Induced Rapid Ventricular Myocardial Capture Demonstrated by Pacemaker Intracardiac Electrograms
}

\author{
MICHAEL CAO, M.D., JEROLD S. SHINBANE, M.D., JEFFREY M. GILLBERG, M.S.* \\ and LESLIE A. SAXON, M.D. \\ From the Keck School of Medicine, University of Southern California, Los Angeles, California; and *Medtronic Inc., \\ Minneapolis, Minnesota, USA
}

\begin{abstract}
Taser-Induced Myocardial Capture. Introduction: A Taser weapon is designed to incapacitate violent individuals by causing temporary neuromuscular paralysis due to current application. We report the first case of a Taser application in a person with a dual-chamber pacemaker demonstrating evidence of Taser-induced myocardial capture.

Methods and Results: Device interrogation was performed in a 53-year-old man with a dual-chamber pacemaker who had received a Taser shot consisting of two barbs delivered simultaneously. Assessment of pacemaker function after Taser application demonstrated normal sensing, pacing thresholds, and lead impedances. Stored event data revealed two high ventricular rate episodes corresponding to the exact time of the Taser application.

Conclusions: This report describes the first human case of ventricular myocardial capture at a rapid rate resulting from a Taser application. This raises the issue as to whether conducted energy devices can cause primary myocardial capture or capture only in association with cardiac devices providing a preferential pathway of conduction to the myocardium. (J Cardiovasc Electrophysiol, Vol. 18, pp. 876-879, August 2007)
\end{abstract}

conducted energy devices, intracardiac electrograms, myocardial capture, pacemaker, Taser

\section{Introduction}

Conducted energy devices (CED) are used by law and military enforcement officials to incapacitate individuals by causing temporary neuromuscular paralysis. The most commonly used CED is the Taser (TASER International, Scottsdale, AZ, USA). More than a quarter million Taser devices have been sold. ${ }^{1}$ A CED is designed to subdue violent individuals and is considered a nonlethal alternative by law and military enforcement officials. Controversy exists about the safety of the device. The Arizona Republic newspaper reported 167 cases of death following Taser application between September 28, 1999 and January 5, 2006 in the United States and Canada. ${ }^{2}$ We report the first case of Taser application in a patient with a dual-chamber pacemaker demonstrating evidence of Taserinduced cardiac capture.

\section{Methods and Results}

A 53-year-old male with a dual-chamber pacemaker implanted subcutaneously on the left chest (Medtronic Kappa, model KDR901, atrial lead model \#5568, and ventricular lead model \# 4074, Medtronic Inc., Minneapolis, MN, USA) for syncope and sick sinus syndrome presented for evaluation

Mr. Gillberg is an employee of Medtronic Inc. Dr. Saxon is a consultant for Guidant Corporation and Medtronic Inc.

Address for correspondence: Leslie A. Saxon, M.D., Division of Cardiovascular Medicine, Keck School of Medicine, University of Southern California, 1510 San Pablo St \#322 North, HCC I, Los Angeles, CA 90033, USA. Fax: 323-442-6133; E-mail: saxon@usc.edu

Manuscript received 14 February 2007; Revised manuscript received 27 April 2007; Accepted for publication 2 May 2007.

doi: $10.1111 / \mathrm{j} .1540-8167.2007 .00881 . x$
1 week after a CED shot. The individual received a Taser shot while running to avoid capture in a prison. The Taser shot consisted of two barbs delivered simultaneously (Taser model X26). The Taser shot struck the man on the right chest. He was temporarily immobilized with subsequent recovery, and according to records, did not suffer any immediate observable adverse effects.

The patient presented 1 week later for medical evaluation due to nonspecific chest pain. Device interrogation revealed normal pacemaker function, defined as normal atrial sensing 4.0-5.0 mV, atrial threshold 2.0V @ $0.06 \mathrm{~ms}$, atrial impedance $732 \mathrm{ohms}$, ventricular sensing $23-30 \mathrm{mV}$, ventricular threshold2.0V @ $0.09 \mathrm{~ms}$, and ventricular lead impedance $659 \mathrm{ohms}$. Both leads were programmed in the bipolar mode (Fig. 1). There was no prior pacemaker interrogation available.

Interrogation of the pacemaker stored events revealed two ventricular high rate episodes that corresponded to the exact time of the Taser barb applications (Figs. 2-4). The stored EGM for both of these episodes was the summed EGM, which combines the atrial and ventricular EGM signals into a single tracing. Prior to the ventricular capture, the atrial rhythm was sinus tachycardia (cycle length $460-476 \mathrm{msec}$ ). This corresponds to the left side of Figure 2. The atrial cycle length is listed on the bottom panel and atrial sensing is indicated by AS on the top panel. The ventricular sensed events are annotated in the middle panel. Next, atrial sensing is suspended due to triggering of postventricular atrial blanking (PVAB) on each ventricular sense (and refractory sense) event and activation of the noise reversion feature, which likely reduced atrial sensitivity during increased high frequency noise caused by Taser pulses (Fig. 2A). Ventricular safety pacing occurs after each atrially paced event due to sensed ventricular events (no markers) within $110 \mathrm{~ms}$ of the delivered atrial pace. The first ventricular high rate episode lasted 5 seconds. 
Pacemaker Programming:

$\begin{array}{ll}\text { Modes: } & \\ \text { Mode: } & \text { DDDR } \\ \text { Mode Switch: } & \text { On } \\ \text { Detection Rate: } & 170 \mathrm{bpm} \\ & \\ \text { Rate: } & \\ \text { Lower Rate Limit: } & 60 \mathrm{bpm} \\ \text { Upper Tracking Rate: } & 130 \mathrm{bpm} \\ \text { Upper Sensor Rate: } & 130 \mathrm{bpm} \\ & \\ \text { AV Intervals: } & \\ \text { Paced AV Interval: } & 230 \mathrm{~ms} \\ \text { Sensed AV Interval: } & 230 \mathrm{~ms} \\ \text { Rate Adaptive AV Interval: } & \text { Off } \\ \text { Search A-V Interval: } & 120 \mathrm{~ms}\end{array}$

Figure 1. Programmed parameters of the dual-chamber pacemaker Medtronic Kappa KDR 901.

After the second ventricular safety paced beat (Fig. 2, right side on the panel), high-rate ventricular sensing continued at cycle length 281-290 msec and at end of the application of Taser energy, a single, long ventricular interval (648 ms) is seen upon resumption of atrial and ventricular sensing with a rate consistent with the pre-Taser rate (cycle length $453 \mathrm{~ms}$ ). Figure 3 is a magnified illustration of the right side of Figure 2 and clearly shows $66 \mathrm{msec}$ high-frequency Taser noise that corresponds to the manufacturer's specified pulse per second (15 pps). In Figure 3, the last Vs EGM during Taser application is traced and superimposed on each prior Vs event (bold, arrows). The EGM from the last Vs during Taser application is superimposed on each prior Vs event to show that the disturbance of the high-frequency Taser signal is consistent with a modulation of the signal by a repeating $R$ wave with a morphology different from the intrinsic $\mathrm{R}$ wave (right side of Fig. 3). Figure 4 shows the EGM stored during the second application of Taser energy within a minute of the first application. The characteristics seen in this tracing are similar to the first Taser application shown in Figures 2 and 3: (1) High-rate ventricular sensing (cycle length 234-
$304 \mathrm{~ms}$ ) with suspension of atrial sensing, (2) modulation of high-frequency Taser pulses (15 pps) at each Vs event, (3) single. long ventricular interval (531 ms) after Taser energy stops, and (4) post-Taser resumption of atrial and ventricular sensing at a rate similar to the pre-Taser cardiac rate.

\section{Discussion}

Taser guns fire small dart-like barbs toward the patient that serve as electrodes with attached metal wires. They are propelled by small gas charges. The maximum firing range is $10 \mathrm{~m}$. The voltage temporarily disrupts nerve and muscle function, resulting in local paralysis, and does not have to penetrate the skin to be effective. ${ }^{1}$ The Taser causes temporary incapacitation by what the manufacturer refers to as a "shaped pulse" technology, which allows stimulation energy to penetrate through clothing via an ionized air from the "arc" phase. The Taser generates an arcing voltage of 50,000 volts with a pulse duration of 100 microseconds and a pulse rate of 19 pps. Certain Taser X26 models decrease the pulse rate from the initial 19 to 15 pps after 2 seconds. The pulses occur in a 5-second cycle. Energy is dispersed to penetrate up to 2 inches of clothing or other barriers to the skin. Incapacitation is achieved via tetanic contractions. ${ }^{4}$

Studies in swine have evaluated the risk of inducing ventricular arrhythmias with Taser application. McDaniel et al. determined that the voltage delivered with a CED discharge to induce ventricular fibrillation was 15-42 times the charge of the standard neuromuscular incapacitating device. ${ }^{5}$ As many individuals who receive Taser applications have used illicit drugs, studies have evaluated the effects of cocaine on ventricular fibrillation thresholds. Lakkireddy et al. determined that CEDs did not cause ventricular fibrillation and that cocaine actually increased the safety margin by $50-100 \%$ in swine. ${ }^{6}$ Interestingly, Nanthakumar et al. applied a total of 150 CED discharges directly to the heart of six swine, where 74 of these discharges resulted in ventricular stimulation and capture. ${ }^{7}$

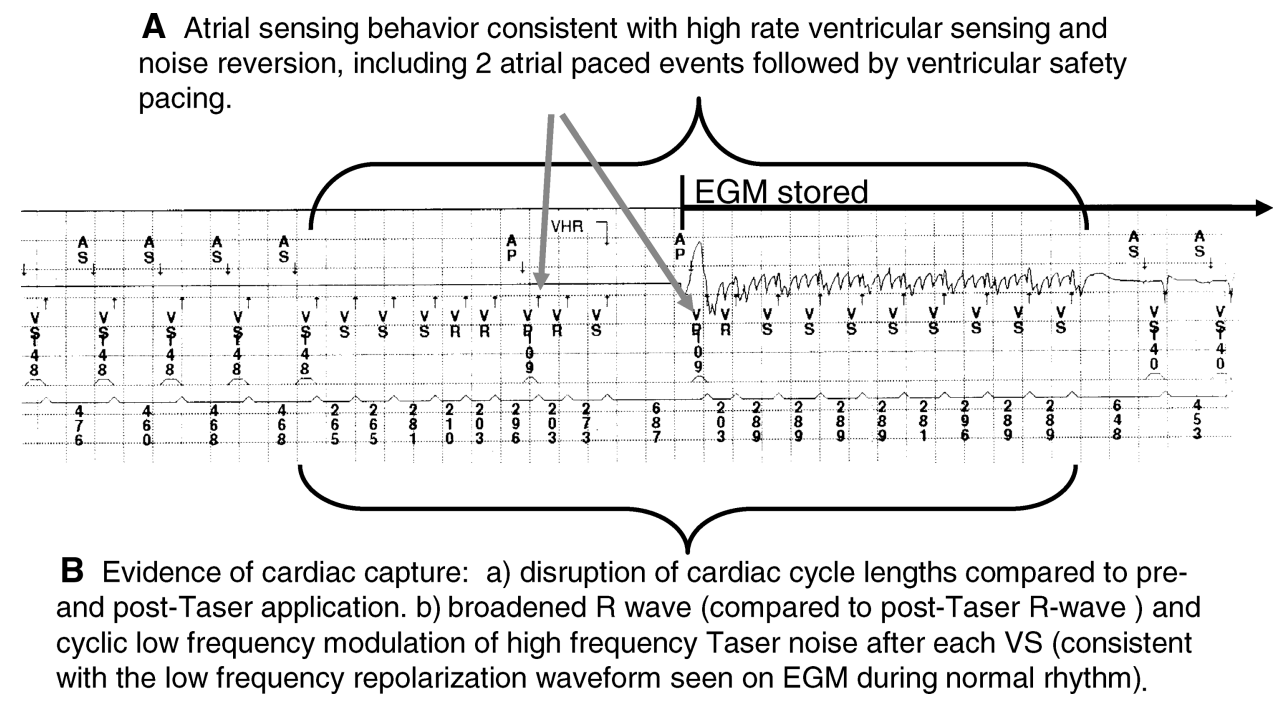

Figure 2. Taser application demonstrates evidence of pacemaker-induced cardiac capture as manifested by: (1) High-rate ventricular sensing (cycle length 203-289 ms) with suspension of atrial sensing (2) cyclic, low-frequency modulation of high frequency noise (Taser pulses) during ventricular sensing (3) single, long ventricular interval (648 msec) after Taser energy stops, and (4) post-Taser resumption of atrial and ventricular sensing at rate similar to pre-Taser cardiac rate. The EGM recorded was the summed EGM, which is a combination of atrial and ventricular EGMs into a single tracing. 


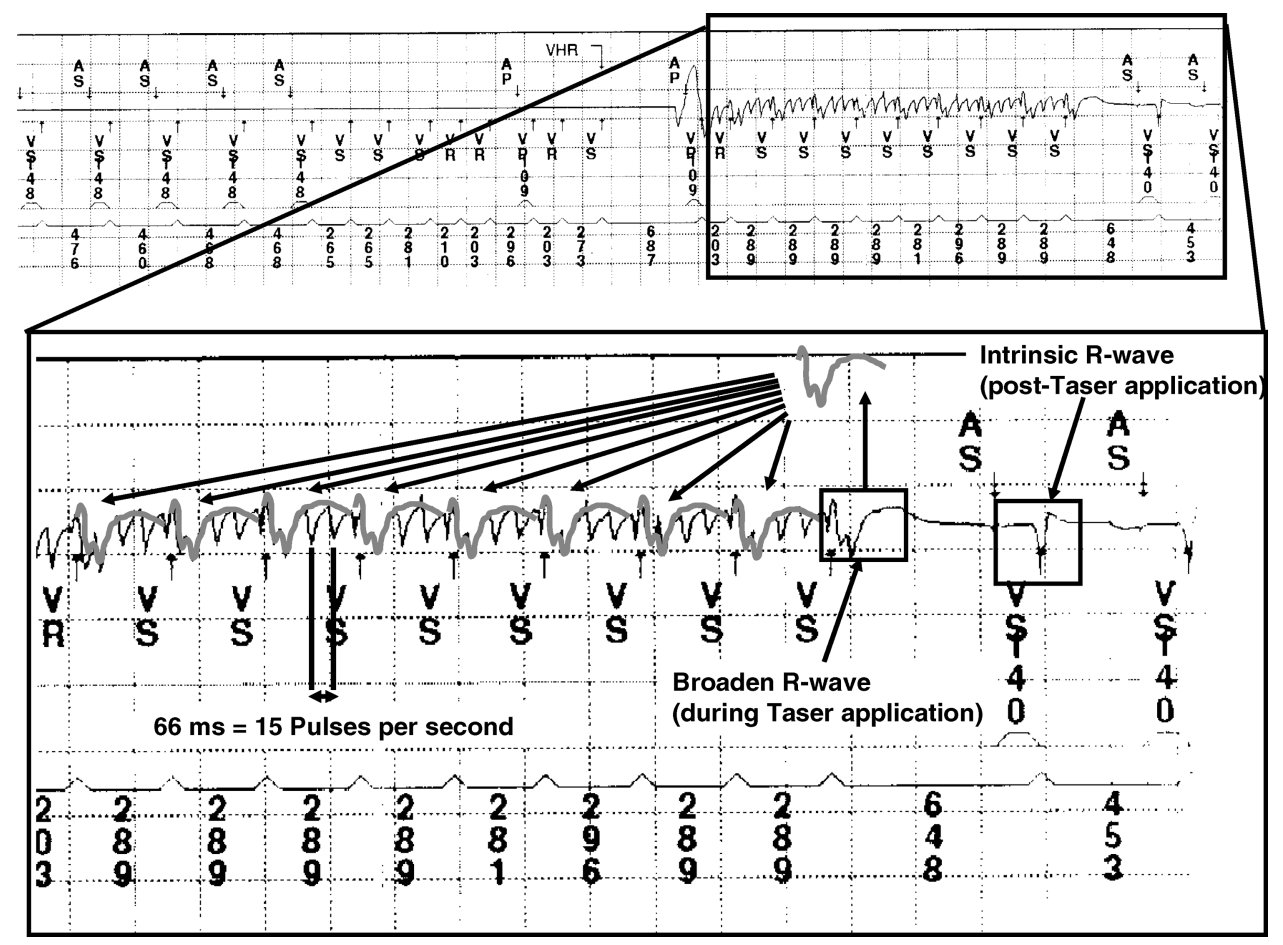

Figure 3. Magnified summed EGM tracing stored during Taser application. The high-frequency Taser pulses (15 pps, 66 ms) are labeled on the tracing. The EGM from the last Vs during Taser application is superimposed on each prior Vs event, showing that the disruption of the high-frequency Taser signal is consistent with a modulation of the signal by a repeating $R$ wave with morphology different than the intrinsic $R$ wave (right side of figure).

There have been few human reports of Taser applications affecting an individual's cardiac rhythm. Haegli et al. reported the effects of a Taser application in a patient with an internal cardiac defibrillator. ${ }^{8}$ The defibrillator sensed high- frequency, high-amplitude electrical signals at an interval of $138-275$ msec. This was detected as ventricular fibrillation; however, no shock was delivered because of a lack of "reconfirmation" and limited exposure time. In an editorial, Marine

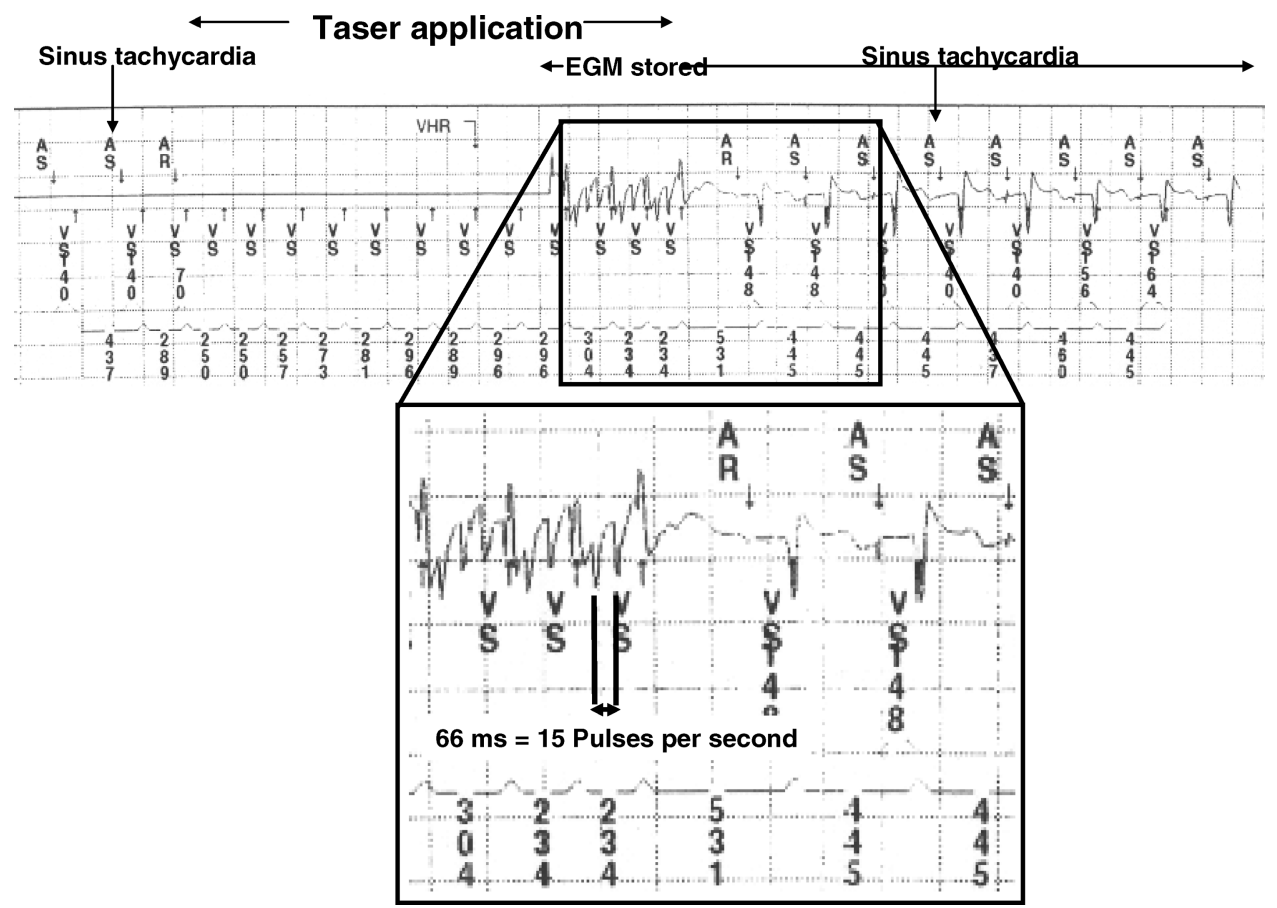

Figure 4. Stored summed EGM during second Taser application (within a minute of first application shown in Figures 2 and 3). The EGM tracing during Taser is magnified and shows Taser energy at 15 pps $(66 \mathrm{~ms})$ and EGM signal and cardiac cycle length disruption similar to first application. See text for details. 


\begin{tabular}{lclc}
\hline Pacemaker Programming & & & \\
\hline Modes & DDDR & Refractory/Blanking & PVARP \\
Mode: & On & Minimum PVARP & Auto \\
Mode switch & $170 \mathrm{bpm}$ & PVAB & $180 \mathrm{~ms}$ \\
Detection rate & & Ventricular refractory & $230 \mathrm{~ms}$ \\
& & Vent. blanking (after & $28 \mathrm{~ms}$ \\
& & A. pace) & \\
Rate & $60 \mathrm{bpm}$ & PMT intervention & Off \\
Lower rate limit & $130 \mathrm{bpm}$ & PVC response & On \\
Upper tracking rate & $130 \mathrm{bpm}$ & Ventricular safety pacing & On \\
Upper sensor rate & & & \\
AV intervals & $230 \mathrm{~ms}$ & & \\
Paced AV interval & $230 \mathrm{~ms}$ & & \\
Sensed AV interval & Off & & \\
Rate adaptive AV interval & $120 \mathrm{~ms}$ & & \\
Search A-V interval & & & \\
\hline
\end{tabular}

noted that none of the Taser pulses appeared to capture ventricular myocardium. ${ }^{1}$ Kim et al. reported an adolescent who was found to have ventricular fibrillation after a stun gun discharge; however, there were no rhythm strips recorded at the time of the stun gun discharge. ${ }^{9}$ Immobilizing criminals with Taser guns is the increasingly preferred method for law enforcement agencies. ${ }^{4}$ This is a nonlethal alternative to using a firearm. One study compared 218 individuals subdued by police with a firearm versus a Taser gun and found a significant difference in mortality (1.4\% vs $50 \%$ in the firearm group). The three fatalities in the Taser group were due to cardiac arrest and the documented rhythm was asystole. ${ }^{3}$

There are several reports of human volunteers who received Taser application without any cardiac sequelae. ${ }^{10,11}$ There are several studies of the effects of a Taser application on normal hearts. Ho et al. evaluated the effects of Taser application on 66 healthy human subjects. ${ }^{10}$ All subjects underwent electrocardiographic monitoring and serial laboratory assessments. No arrhythmias or laboratory abnormalities were detected. Levine et al. evaluated 20 healthy human police officers who received a Taser application. ${ }^{11}$ Electrocardiographic monitoring resulted in sinus tachycardia. No previous report has documented direct intracardiac monitoring during a Taser application with the induction of ventricular capture.

Kornblum et al. performed autopsies in 16 individual deaths associated with Taser applications. ${ }^{12}$ They identified one directly attributable to Taser application. ${ }^{12}$ In an editorial, Allen, a medical examiner involved with several of the death investigations, commented that up to nine causes of death were associated with Taser. ${ }^{13}$

The potential mechanism of CED myocardial capture in association with cardiac devices requires further investigation. Sweesy et al. discuss the various device responses of electromagnetic interference in cardiac devices. ${ }^{14}$ Generally, device responses to electromagnetic interference can include: no effect, oversensing, noise reversion, circuitry damage, induced current or sensor-driven pacing, incorrect diagnostics, or unintended device reprogramming. After the Taser shot, the discharge travels from one Taser barb to the other, following the path of least resistance. An additional electric field outside of the immediate proximity of the barbs is generated, stimulating a sufficient volume of skeletal muscle through indirect stimulation of the motor nerves. ${ }^{15}$ It is likely that the close proximity of the Taser barbs (right chest) to the pacemaker (left chest) subjected the pacemaker generator and leads to the Taser-generated electric field. The Taser discharge can potentially conduct directly to the leads or via the pacemaker through the leads and then to the heart. Preliminary analysis by engineers from the pacemaker manufacturer indicates that there is the possibility of an electrical pathway from the pacemaker housing the electrodes in the heart if there is a large voltage on the housing relative to the electrodes in the heart. The fact that the pacemaker did not oversense the Taser artifact and yet sensed the $\mathrm{R}$ waves is likely attributed to the bandpass-filtering and thresholding techniques used in the sense amplifier to reject noise. Whether a device leads alone (without connection to the pacemaker device itself) actually increases susceptibility to cardiac capture is unknown at this time. As structurally abnormal hearts may be further compromised by rapid ventricular stimulation, Taser discharges could pose additional risk to those individuals who have either a pacemaker or internal cardiac defibrillator.

\section{Conclusion}

This report describes the first human case of ventricular myocardial capture at a rapid rate resulting from a Taser current application. This raises the issue as to whether CEDs such as the Taser can cause primary myocardial capture or capture only in association with cardiac devices providing a preferential pathway via the pacing system to the myocardium. Further investigation is required to understand the effects of CEDs on people with cardiac devices.

\section{References}

1. Marine JE: Stun guns: A new source of electromagnetic interference for implanted cardiac devices. Heart Rhythm 2006;3:342-344.

2. Anglen R: 167 cases of death following stun-gun use. Arizona Republic January 5, 2006.

3. Ordog GJ, Wasserberger J, Schlater T, Balasubramanium S: Electronic gun (Taser) injuries. Ann Emerg Med 1987;16:73-78.

4. Taser. http://www2.taser.com/products/consumers/Pages/TASERX26C. aspx. http://www.taser.com

5. McDaniel WC, Stratbucker RA, Nerheim M, Brewer JE: Cardiac safety of neuromuscular incapacitating defensive devices. Pacing Clin Electrophysiol 2005;28(Suppl 1):S284-287.

6. Lakkireddy D, Wallick D, Ryschon K, Chung M, Butany J, Martin D, Saliba W, Kowalewski W, Natale A, Tchou P: Effects of cocaine intoxication on the threshold for stun gun induction of ventricular fibrillation. J Am Coll Cardiol 2006;48:805-811.

7. Nanthakumar K, Billingsley I, Masse S, Dorian P, Cameron D, Chauhan V, Downar E, Sevaptsidis E: Cardiac electrophysiological consequences of neuromuscular incapacitating device discharges. J Am Coll Cardiol 2006; $48: 798-804$.

8. Haegeli LM, Sterns LD, Adam DC, Leather RA: Effect of a Taser shot to the chest of a patient with an implantable defibrillator. Heart Rhythm 2006;3:339-341.

9. Kim PJ, Franklin WH: Ventricular fibrillation after stun-gun discharge. N Engl J Med 2005;353:958-959.

10. Ho JD, Miner JR, Lakireddy DR, Bultman LL, Heegaard WG: Cardiovascular and physiologic effects of conducted electrical weapon discharge in resting adults. Acad Emerg Med 2006;13:589-595.

11. Levine S, Sloane C, Chan T, Vilke G, Dunford J: Cardiac monitoring of subjects exposed to the Taser. Acad Emerg Med 2005;12:S71.

12. Kornblum RN, Reddy SK: Effects of the Taser in fatalities involving police confrontation. J Forensic Sci 1991;36:434-438.

13. Allen TB: Discussion of effects of the Taser in fatalities involving police confrontation. J Forensic Sci 1992;37:956-958.

14. Sweesy MW, Holland JL, Smith KW: Electromagnetic interference in cardiac rhythm management devices. AACN Clin Issues 2004;15:391403 .

15. Panescu D, Kroll MW, Efimov IR, Sweeney JD: Finite element modeling of electric field effects of Taser devices on nerve and muscle. Proceedings of the 28th IEEE EMBS Annual International Conference, 2006, 1277-1279. 